\title{
Development of metal sulfide-poly (3-octylthiophene) composite LB multilayers
}

\author{
V. Vidya ${ }^{\text {a }}$, S. Ambily ${ }^{\text {a }, ~ S . N . ~ N a r a n g ~}{ }^{\text {a }}$, S. Major ${ }^{\text {a,* }}$, S.S. Talwar ${ }^{\text {b }}$ \\ ${ }^{a}$ Department of Physics, Indian Institute of Technology, Bombay, Mumbai 400 076, India \\ ${ }^{\mathrm{b}}$ Department of Chemistry, Indian Institute of Technology, Bombay, Mumbai 400 076, India
}

\begin{abstract}
Langmuir-Blodgett (LB) technique has been used to deposit composite multilayers of poly(3-octylthiophene) with cadmium arachidate (POT-CdA), zinc arachidate (POT-ZnA) and copper arachidate (POT-CuA). These composite multilayers were used as precursors to develop the respective semiconducting $\mathrm{CdS}, \mathrm{ZnS}$ and $\mathrm{Cu}_{2} \mathrm{~S}$ nanoclusters in the POT-arachidic acid (POT-AA) matrix. The formation of sulphide nanoclusters in the multilayer was determined by FTIR and UV-Vis spectroscopy. X-ray reflectivity measurements showed a drastic reduction in the layered structural order on sulphide formation. Single layer LED structures were fabricated using POT-CdA, CdS-POT-AA and ZnS-POT-AA composites as active layers. The Electoluminescence peaks from these structures are attributed to POT and nanoclusters of $\mathrm{CdS}$ and $\mathrm{ZnS}$, respectively. The electroluminescent devices with nanoclusters containing emitter layers exhibited low turn-on voltage $\sim 5 \mathrm{~V}$. Blue electroluminescence was observed at room temperature from FTO/ZnS-POT-AA/Al devices.
\end{abstract}

Keywords: Poly(3-octylthiophene); LB films; Nanoclusters; LED

\section{Introduction}

Organic light emitting devices have recently attracted a great deal of attention exhibiting features like high quantum efficiency and colour tunability [1-5]. These devices involve simple chemical processing routes and have the potential for large area display applications. The more recent approach of developing hybrid organic/inorganic optoelectronic devices [6,7] combines the advantages of both organic and inorganic semiconducting systems. Semiconducting nonocluster-polymer composites for example, provide a wide selection range for emitter and carrier transport materials, in addition to the mechanical strength, flexibility and surface passivation behaviour of the polymers. The applications of semiconducting nanoclusters or quantum dots of II-VI compounds such as CdSe [7-10], CdS [11] and $\mathrm{ZnS}[12,13]$ embedded in polymer matrix have been demonstrated in light emitting diodes [7-9,12,13], photovoltaics [10] and non-linear optics [11]. Langmuir-Blodgett (LB) and self assem- 
bly techniques are important routes to the development of organized layered structures of such nanocomposite systems with specially designed architecture and functionality [14]. In particular, post deposition treatment of LB multilayers is a simple approach which has been used to grow chalcogenide semiconducting nanoclusters in organic fatty acid as well as polymeric matrix [1519]. There is however a need to explore this approach by extending it to a variety of semiconducting nanocluster and polymeric systems and understand the kinetics of formation, growth and organization of the nanoclusters as well as the overall structure of the nanocomposite multilayers.

Poly(3-alkylthiophenes) are one of the most promising conducting polymers known for their interesting electronic and optical properties combined with easy processibility and chemical stability. There has recently been considerable interest toward their application in electroluminescent and photovoltaic devices [20,21]. In the present work, the composite monolayer methodology [22,23], in which a non amphiphilic polymer is mixed with a suitable metal ion containing amphiphilic molecule, has been used to develop composite LB multilayers of poly(3-octylthiophene) (POT) with cadmium arachidate (POT-CdA), zinc arachidate (POT-ZnA) and copper arachidate (POT$\mathrm{CuA}$ ). The composite multilayers were subjected to post deposition $\mathrm{H}_{2} \mathrm{~S}$ exposure to grow $\mathrm{CdS}$, $\mathrm{ZnS}$ and $\mathrm{Cu}_{2} \mathrm{~S}$ nanoclusters within the polythiophene-arachidic acid (POT-AA) composite multilayers. The formation of semiconducting nanoclusters in the POT-AA matrix was studied by FTIR and UV-VIS spectroscopies and X-ray reflectivity was used to study the layered structure of the multilayers. Electroluminescence measurements were carried out on nanocluster/polymer based single layer LED structures.

\section{Experimental}

The POT based composite LB multilayers were deposited using a KSV 3000 system. Deionised and Millipore ultrafiltered water (resistivity 18.2 $\mathrm{M} \Omega, \mathrm{pH}$ 5.7) was used as the subphase. A mixture of POT and Arachidic acid (1:1 by weight) was spread on the subphase containing $\mathrm{CdCl}_{2}, \mathrm{ZnCl}_{2}$ and $\mathrm{CuCl}_{2}$ salts $\left(4 \times 10^{-4} \mathrm{M}\right)$ at a subphase $\mathrm{pH}$ of 6.4 to deposit POT-CdA and POT-CuA multilayers. POT-ZnA multilayers were transferred at a higher subphase $\mathrm{pH} \sim 7.5$ to ensure the packing of arachidate molecules perpendicular to the layer plane as reported earlier [24]. All the multilayers were transferred at a constant surface pressure of $18 \mathrm{mN} \mathrm{m}^{-1}$, a subphase temperature of $10{ }^{\circ} \mathrm{C}$ and a dipping speed of $3 \mathrm{~mm} \min ^{-1}$ on quartz, $\mathrm{CaF}_{2}$ and fluorine doped tin oxide (FTO) substrates. Hydrogen sulfide gas exposure of the multilayers was carried out in a closed glass chamber at room temperature and at atmospheric pressure. FTIR measurements were recorded using a Nicolet (Impact-400) spectrophotometer. The UV-Vis spectra were obtained using a Shimadzu 160A spectrophotometer. X-ray reflectivity measurements were carried out with a Philips PW-1710 powder diffractometer using $\mathrm{Cu}$ target. The diffraction patterns were obtained in the $2 \theta$ range of $4-20^{\circ}$. Electroluminescence was studied with a Jobin Yvon spectrometer consisting of a monochromator and photomultiplier tube. The spectral range of the monochromator was from 200 to $750 \mathrm{~nm}$. The photomultiplier tube is installed at the exit side of the monochromator and has a single channel detector used for low light spectroscopic measurements from 200 to $1100 \mathrm{~nm}$. The sample for EL measurement was placed directly in front of the spectrometer without any collimating optics.

\section{Results and discussion}

Composite POT-AA monolayers were transferred from the metal $(\mathrm{Cd}, \mathrm{Zn}$ and $\mathrm{Cu})$ ion containing subphase to form POT-CdA, POT-ZnA and POT-CuA multilayers under the conditions described above. The transfers were Y-type with near unity transfer ratios and 25 monolayers were transferred in all the three cases.

The FTIR spectra of the as-deposited and $\mathrm{H}_{2} \mathrm{~S}$ exposed composite polymer are shown in Fig. 1. The as-deposited POT-CdA and POT-ZnA multilayers show a strong absorption peak around 
$1545 \mathrm{~cm}^{-1}$ (curves a and $\mathrm{b}$, respectively). In the case of POT-CuA, (curve c) this peak is shifted to $\sim 1590 \mathrm{~cm}^{-1}$. These strong peaks correspond to asymmetric carbonyl stretching vibration of the carboxylate group, indicating that all the transferred multilayers were present in the form of arachidate salt. The peak position in case of POT$\mathrm{CuA}$ is characteristic of transition metal arachidates [25]. On exposure to $\mathrm{H}_{2} \mathrm{~S}$ (typically $\sim 1-2$ $\mathrm{h}$ ), the peak corresponding to arachidate salt disappears in all the cases and a strong absorption peak at $\sim 1700 \mathrm{~cm}^{-1}$ (curves $(\mathrm{d}-\mathrm{f})$ ) due to carbonyl stretching vibration of the carboxylic acid group appears, indicating complete conversion of arachidic salt to arachidic acid. The chemical conversion of the salt to acid is indicative of the formation of the respective metal sulfides in the multilayers. The FTIR spectra show no changes on longer exposures $(>2 \mathrm{~h})$ indicating a saturation behaviour of the chemical reaction.

The UV-Vis absorption spectra from POTCdA, POT-ZnA and POT-CuA multilayers both in the as deposited and $3 \mathrm{~h} \mathrm{H}_{2} \mathrm{~S}$ exposed states are shown in Fig. 2. In all the three cases, the absorption spectra in the as-deposited state show a broad peak at $\sim 500 \mathrm{~nm}$, characteristic of POT, confirming the presence of the polymer in the composite multilayers [22]. In the case of POTCdA multilayer exposed to $\mathrm{H}_{2} \mathrm{~S}$ gas (Fig. 2(a)), an enhanced absorbance below $500 \mathrm{~nm}$ is observed. The increase in absorbance is attributed to the formation of $\mathrm{CdS}$ nanoclusters within the composite polymer matrix. Though the exact absorption onset of $\mathrm{CdS}$ is overshadowed by the presence of the strong POT peak, it is clearly blue shifted compared to the absorption edge of bulk

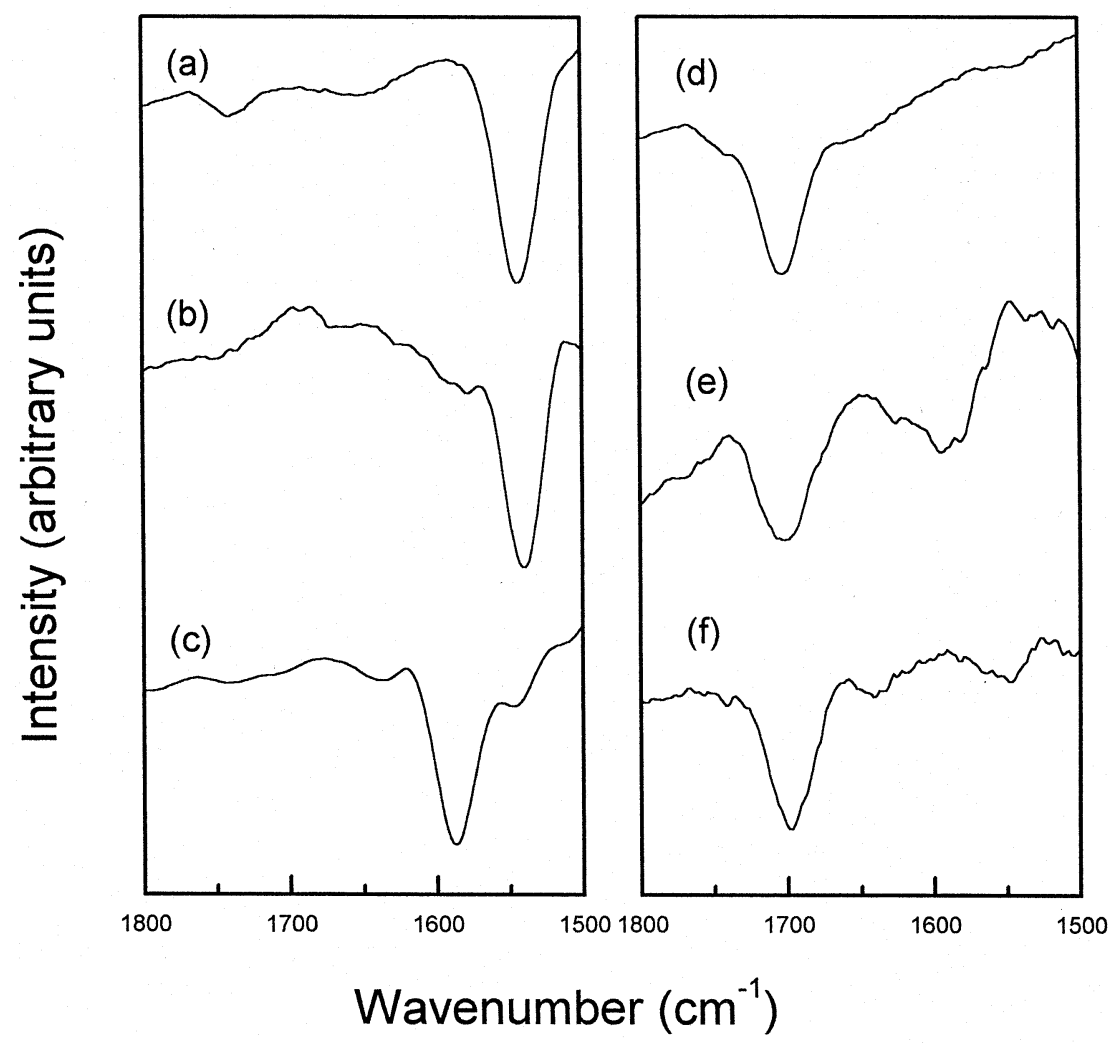

Fig. 1. FTIR spectra of (a) POT-CdA (b) POT-ZnA and (c) POT-CuA multilayers in the as deposited states shows the asymmetrical carbonyl stretching vibration of carboxylate group. The corresponding spectra (d, e, f) after $2 \mathrm{~h}_{2} \mathrm{~S}$ exposure show the carbonyl stretching vibration $\sim 1700 \mathrm{~cm}^{-1}$ of the carboxylic acid group in all the three cases. 


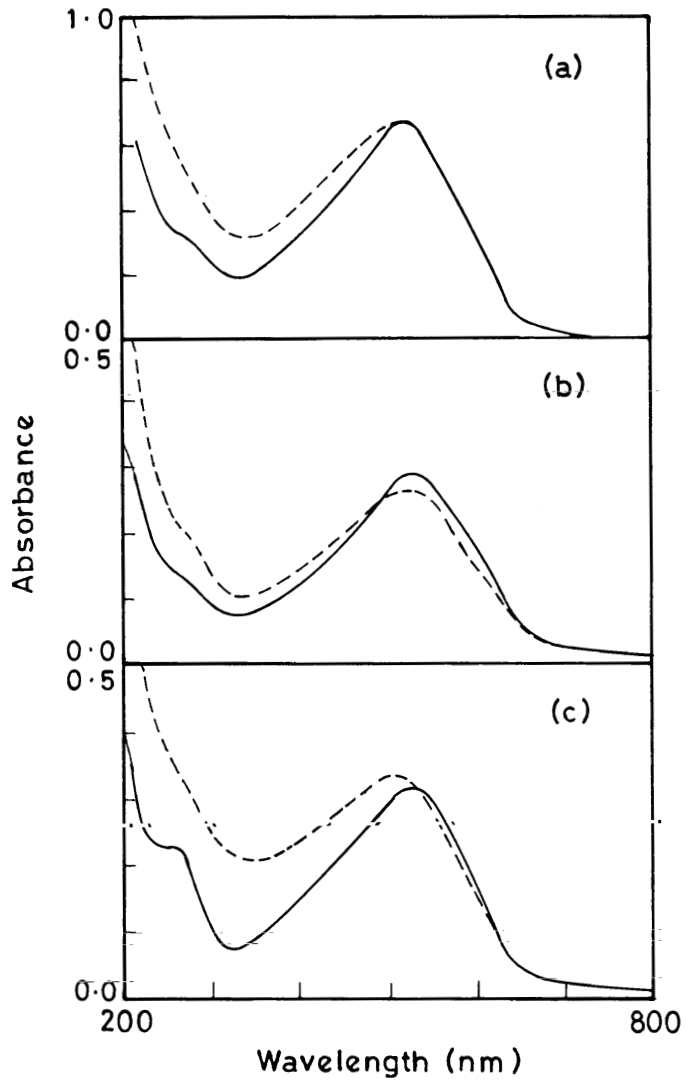

Fig. 2. UV-Vis spectra of (a) POT-CdA, (b) POT-ZnA and (c) POT-CuA multilayers in the as deposited (-) and $\mathrm{H}_{2} \mathrm{~S}$ exposed (---- -) states. The enhanced absorption at $<500 \mathrm{~nm}$ indicates semiconducting sulphide nanocluster formation in all the three cases.

CdS $(515 \mathrm{~nm})$. In the case of POT-ZnA exposed to $\mathrm{H}_{2} \mathrm{~S}$, an enhanced absorption is observed below $480 \mathrm{~nm}$. In this case also, the exact absorption onset could not be ascertained due to the presence of the strong POT peak. The spectrum also exhibits a small peak $\sim 280 \mathrm{~nm}$, which is attributed to the excitonic band of $\mathrm{ZnS}$ suggesting the formation of $\mathrm{ZnS}$ nanoclusters [26]. In the case of POT-CuA, on exposure to $\mathrm{H}_{2} \mathrm{~S}$, the absorbance below $500 \mathrm{~nm}$ increases significantly. The increase in absorbance is attributed to formation of $\mathrm{Cu}_{2} \mathrm{~S}$ nanoparticles in the AA matrix. It is interesting to note that the absorption onset in this case is considerably shifted from the bulk value of 1022 nm.
The layered structure of the POT-CdA, POT$\mathrm{ZnA}$ and POT-CuA multilayers have been investigated using X-ray reflectivity scans shown in Fig. 3. The POT-CdA multilayers (a) show well defined (00l) Bragg peaks indicating the presence of a well formed layer structure. The average bilayer period determined from the position of the Bragg peaks was $55 \AA$, which corresponds to alkyl chains packed nearly perpendicular to the substrate. The POT-ZnA multilayers (b) also exhibited a nearly similar layered structure with a bilayer period of $55 \AA$ A. However, the Bragg peaks in this case are relatively weaker and broad indicating lesser degree of layered structural order as compared to POT-CdA multilayer. In comparison, the X-ray reflectivity scan of the as-deposited POT-CuA (c) multilayer however showed a much reduced structural order. The bilayer period of $\sim 53 \AA$ obtained in this case is attributed to the tilting of alkyl chains in these multilayers. The

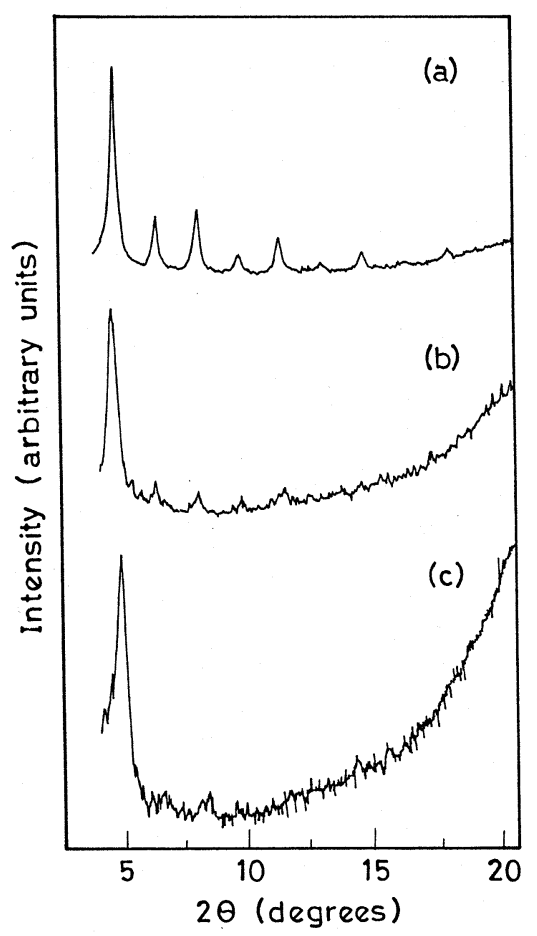

Fig. 3. X-ray reflectivity from (a) POT-CdA, (b) POT-ZnA and (c) POT-CuA composite multilayers. The POT-CdA multilayer shows high interlayer order compared the other two composite multilayers. 
$\mathrm{H}_{2} \mathrm{~S}$ exposed films in all the three cases did not exhibit any Bragg peak indicating a drastic reduction in layered structural order on $\mathrm{H}_{2} \mathrm{~S}$ exposure.

Electroluminescence (EL) studies were carried out at room temperature on $\mathrm{FTO} /$ composite polymer/Al single layer LED structures. Composite multilayers of POT-CdA and POT-ZnA were transferred on fluorine doped tin oxide substrates (sheet resistance $<25 \Omega$ ). The composite multilayers were subsequently exposed to $\mathrm{H}_{2} \mathrm{~S}$ to form the respective metal sulfide nanoclusters. Aluminium top electrodes were vacuum evaporated at a pressure $<10^{-5}$ mbar through a teflon mask. The typical area of the circular electrodes was $\sim 4$ $\mathrm{mm}^{2}$. EL measurements were carried out in forward bias condition with FTO as the positive electrode. The EL spectrum from the FTO/POT$\mathrm{CdA} / \mathrm{Al}$ structure was obtained at a turn on voltage of $\sim 15 \mathrm{~V}$ and is shown in Fig. 4(a). The EL peak $\sim 580 \mathrm{~nm}$ along with a shoulder at $\sim 600 \mathrm{~nm}$ is attributed to poly(octyl-thiophene) [27-29]. The position of the EL peak is however blueshifted from the reported EL peak $\sim 660 \mathrm{~nm}$ for polyalkylthiophenes [28,29]. This may be due to the difference in distribution of conjugation lengths. The EL spectra from FTO/CdS-POT$\mathrm{AA} / \mathrm{Al}$ and FTO/ZnS-POT-AA/Al structures are shown in Fig. 4(b) and (c) respectively and exhibit significantly different features. The $\mathrm{Cu}_{2} \mathrm{~S}$-POT-AA based structure did not produce reproducible junctions and was not studied by EL measurements. In the case of CdS-POT-AA based structure, a broad EL peak $\sim 530 \mathrm{~nm}$ is seen which appears to arise from the presence of CdS nanoclusters within the composite polymer, as it is in a region where luminescence from $\mathrm{CdS}$ nanoparticles is usually observed [30]. The EL spectrum from the ZnS-POT-AA based structure in comparison shows a sharp blue emission peaked at $\sim 440 \mathrm{~nm}$ which is attributed to $\mathrm{ZnS}$ nanoparticles in the polymer matrix [13,31]. It is interesting to note that in both the later cases, the turn-on voltage was found to be considerably reduced to $\sim 5 \mathrm{~V}$ as compared to the POT-CdA based LED structure. More detailed investigations of these composite polymer based LED structures are presently in progress.
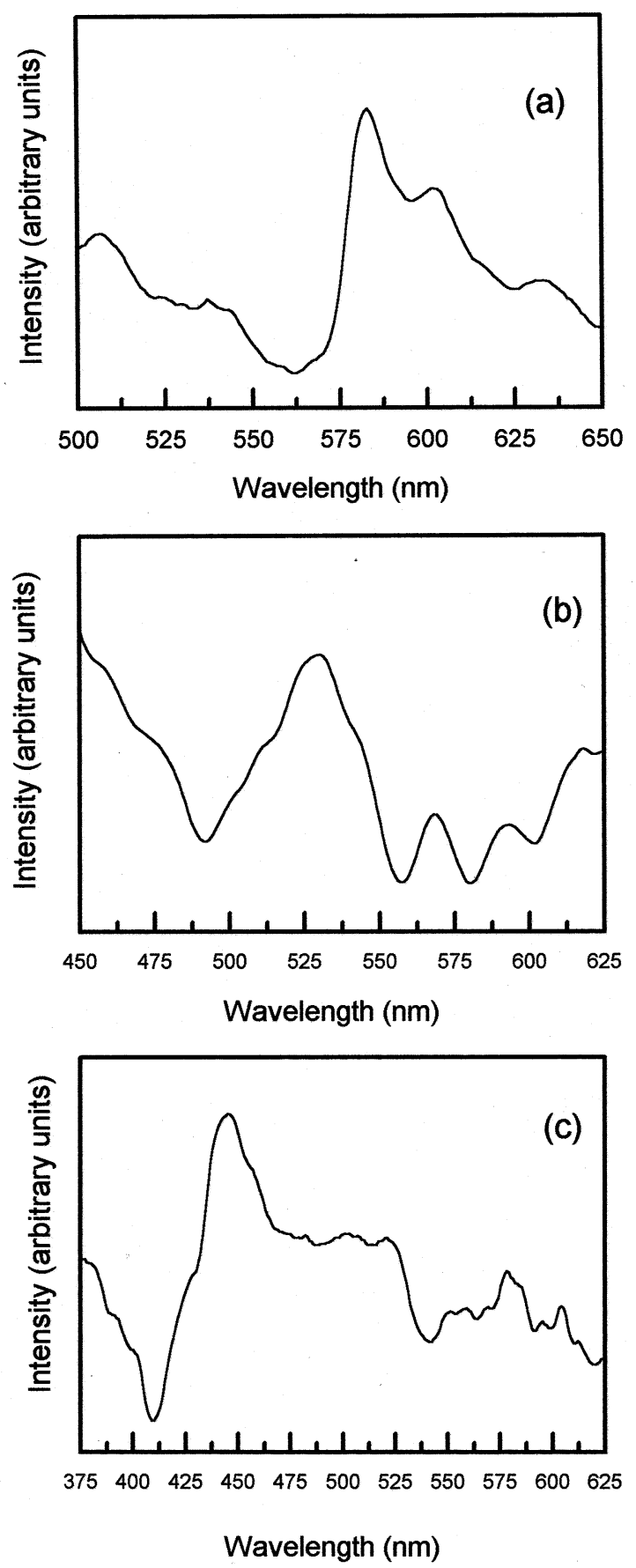

Fig. 4. Room temperature EL spectra from (a) FTO/POT$\mathrm{CdA} / \mathrm{Al}$ (b) FTO/POT-CdS AA/Al and (c) FTO/POT-ZnS$\mathrm{AA} / \mathrm{Al}$ single layer LED structures. 


\section{Conclusions}

Using composite monolayer methodology, POTCdA, POT-ZnA and POT-CuA LB multilayers were deposited and used as precursors to develop the respective semiconducting chalcogenide $(\mathrm{CdS}$, $\mathrm{ZnS}$ and $\mathrm{Cu}_{2} \mathrm{~S}$ ) nanoclusters in POT-AA matrix. The FTIR and UV-VIS absorbance spectra confirmed the presence of semiconducting $\mathrm{CdS}, \mathrm{ZnS}$ and $\mathrm{Cu}_{2} \mathrm{~S}$ nanoclusters in the polymer matrix. The $\mathrm{X}$-ray reflectivity scans showed the presence of a layered structure in all the three cases in the as-deposited state. The structural order in the multilayer is drastically reduced after exposure to $\mathrm{H}_{2} \mathrm{~S}$. The EL measurements on single layer LED structure based on POT-CdA active layer showed EL peak $600 \mathrm{~nm}$ due to POT. The EL peaks from CdS-POT-AA and ZnS-POT-AA based structures could be attributed to the $\mathrm{CdS}$ and $\mathrm{ZnS}$ nanoclusters. The incorporation of semiconducting nanoclusters in the polymer matrix resulted in low turn on voltage $\sim 5 \mathrm{~V}$. Blue luminescence was observed at room temperature from the electroluminescent device having ZnS-POT-AA composite as the emitter layer.

\section{Acknowledgements}

Financial assistance from the Department of Science and Technology, Government of India is gratefully acknowledged. The authors also like to thank Dr R.O. Dusane and S. Patil for their valuable help in electroluminescence measurements.

\section{References}

[1] H. Burroughes, D.D.C. Bradely, A.R. Brown, R.N. Marks, K. Mackay, R.H. Friend, P.L. Brown, A.B. Holumes, Nature (London) 347 (1990) 539.

[2] D. Braun, A.J. Heeger, Appl. Phys. Lett. 58 (1991) 1982.

[3] N.C. Greenham, S.C. Morathi, D.D.C. Bradley, R.H. Friend, A.B. Holmes, Nature (London) 365 (1993) 628.

[4] M.A. Baldo, D.F. O'Brian, Y. You, A. Shouslikov, S. Silbey, M.E. Thompson, S.R. Forrest, Nature (London) 395 (1998) 151.

[5] S. Miyata, H.S. Nalwa, Organic Electroluminescent Materials and Devices, Gorden Breach Science, London, 1997.
[6] V.L. Colvin, M.C. Scblamp, A.P. Alivisatos, Nature (London) 370 (1994) 354.

[7] B.O. Doubousi, M.G. Banwendi, O. Onitsuka, M.F. Rubner, Appl. Phys. Lett. 66 (1995) 1316.

[8] H. Mattoussi, L.H. Radzilowski, B.O. Dabbousi, D.E. Fogg, R.R. Schrock, E.L. Thomas, M.F. Rubner, M.G. Bawendi, J. Appl. Phys. 86 (1999) 4390.

[9] M. Gao, B. Richter, S. Kirstein, H. Möhwald, J. Phys. Chem. B. 102 (1998) 4096.

[10] W.U. Huynh, X. Peng, A.P. Alivisatos, Adv. Mater. 11 (1999) 923.

[11] N. Herron, D.L. Thorn, Adv. Mater. 10 (1998) 1173 and references therein.

[12] Y. Yang, S. Xue, S. Liu, J. Huang, J. Shen, Appl. Phys. Lett. 69 (1996) 377.

[13] J. Huang, Y. Yang, S. Xue, B. Yang, S. Liu, J. Shen, Appl. Phys. Lett. 70 (1997) 2335.

[14] A. Ulman, Organic Thin Films and Surfaces: Directions for the Nineties, Academic Press, London, 1995.

[15] E.S. Smotkin, C. Lee, A.J. Bard, A. Campion, M.A. Fox, T.E. Mallouk, S.E. Webber, J.M. White, Chem. Phys. Lett. 152 (1988) 265.

[16] F. Grieser, D.N. Furlong, D. Scoberg, I. Ichinose, N. Kimizuka, T. Kunitake, J. Chem. Soc., Faraday Trans. 88 (1992) 2207.

[17] B.O. Dabbousi, C.B. Murray, M.F. Rubner, M.G. Bawendi, Chem. Mater. 6 (1994) 216.

[18] A. Dhanbalan, H. Kudrolli, S.S. Major, S.S. Talwar, Solid State Commun. 99 (1996) 859.

[19] A. Dhanabalan, S.S. Talwar, A.Q. Contractor, N.P. Kumar, S.N. Narang, S.S. Major, K.P. Muthe, J.C. Vyas, J. Mater. Sci. Lett. 18 (1999) 603.

[20] P. Barta, J. Sanetra, M. Zagórska, Synthetic Met. 94 (1998) 119.

[21] T. Östergard, A.J. Pal, H. Stubb, J. Appl. Phys. 83 (4) (1998) 2338.

[22] I. Watanabe, K. Hong, M.F. Rubner, Langmuir 6 (1990) 1164.

[23] A. Dhanabalan, R.B. Dabke, S.N. Datta, N. Prasanth Kumar, S.S. Major, S.S. Talwar, A.Q. Contractor, Thin Solid Films 295 (1997) 255.

[24] A. Dhanabalan, N. Prasanth Kumar, S. Major, S.S. Talwar, Thin Solid Films 327-329 (1998) 787.

[25] K. Nakamoto, Infrared Spectra of Inorganic and Coordination Compounds, Wiley, New York, 1963, p. 197.

[26] P.E. Lippens, M. Lanoo, Phys. Rev. B 39 (1989) 10935.

[27] J.L. Sauvajol, D. Chenouni, J.P. Lère-porte, B. Moukala, J. Petrissans, Synthetic Met. 38 (1990) 1.

[28] H. Nishino, G. Yu, A.J. Heeger, T.-A. Chen, R.D. Rieke, Synthetic Met. 68 (1995) 243.

[29] F. Garten, A.R. Schlatmann, R.E. Gill, J. Vrijmoeth, T.M. Klapwijk, G. Hadziioannou, Appl. Phys. Lett. 66 (1995) 2540.

[30] A. Eychmüller, A. Hässelbarth, L. Katsikas, H. Weller, J. Luminesc. $48 / 49$ (1991) 745.

[31] Y. Yang, J. Huang, S. Liu, J. Shen, J. Mater. Chem. 7 (1997) 131. 